\title{
Chiral Cherenkov and chiral transition radiation in anisotropic matter
}

\author{
Kirill Tuchin \\ Department of Physics and Astronomy, Iowa State University, Ames, Iowa 50011, USA
}

(Received 17 October 2018; published 21 December 2018)

\begin{abstract}
A significant contribution to the electromagnetic radiation by a fast electric charge moving in anisotropic chiral matter arises from spontaneous photon radiation due to the chiral anomaly. While such a process, also known as the "vacuum Cherenkov radiation", is forbidden in the QED vacuum, it can occur in chiral matter, where it is more appropriate to call it the "chiral Cherenkov radiation." Its contribution to the radiation spectrum is of order $\alpha^{2}$ compared to $\alpha^{3}$ of the bremsstrahlung. I derive the frequency spectrum and the angular distribution of this radiation in the high energy limit. The quantum effects due to the hard photon emission and the fermion mass are taken into account. The obtained spectra are analyzed in the case the quark-gluon plasma and a Weyl semimetal.
\end{abstract}

DOI: $10.1103 /$ PhysRevD.98.114026

\section{INTRODUCTION}

Electromagnetic radiation by fast particles in chiral matter has a number of unique features that make it a useful tool to investigate the chiral anomaly. The precision of experiments, especially in condensed matter physics, requires a quantitative description of the radiation. It can be derived by applying the standard machinery of the quantum field theory to the effective low energy Maxwell-Chern-Simons Lagrangian (1). The chiral anomaly is encoded in the Chern-Simons term that couples electrodynamics to the pseudoscalar field $\theta$ which reflects the material topological properties. In quark-gluon plasma $\theta$ describes the gluon topological number fluctuations and the associated sphaleron transitions. In Weyl semimetals its gradient is proportional to the splitting between the Weyl nodes. The Chern-Simons term induces a number of novel phenomena such as the chiral magnetic effect and the anomalous Hall effect [1-5].

The first calculation of the electromagnetic radiation due to the $\theta$-term was done in $[6,7]$ who dubbed it the "vacuum Cherenkov radiation" and proposed as a test of the physics beyond the Standard Model. Specifically, it was proposed to be a test of the Lorentz symmetry violation [8-13]. A phenomenon closely related to the vacuum Cherenkov radiation is the chiral transition radiation which is emitted by fast particles crossing the boundary between the chiral matter and vacuum $[14,15]$.

The main goal of this paper is to rederive the photon spectrum using the Lagrangian (1) with the from of the

Published by the American Physical Society under the terms of the Creative Commons Attribution 4.0 International license. Further distribution of this work must maintain attribution to the author(s) and the published article's title, journal citation, and DOI. Funded by SCOAP . $\theta$-field dictated by the applications in condensed matter and nuclear physics. To this end, the calculation method of [14] is employed which allows one to take account of the quantum corrections due to the hard photon emission and the fermion mass. The paper is structured as follows. In Sec. II the effective Lagrangian is introduced. The resulting Maxwell-Chern-Simons equations effectively describe the free electromagnetic field in an anisotropic matter. It is assumed that $c_{A} \theta$, where $c_{A}$ is the anomaly coefficient, is of order unity and thus the Chern-Simons $\tilde{F} F$ term is of the same order of magnitude as the usual $F F$ term of the Maxwell theory. The field is then quantized in Sec. III and its properties, in particular the polarization, are discussed. The radiation rate is computed in Sec. IV in the leading order in the perturbation theory. Since in practical applications fermions are ultrarelativistic, I employ the highenergy (or ultrarelativistic) approximation in Sec. V, which allows me to derive simple expressions for the photon spectra. The main results are given by (37), (41), (42), and (46). Figures 2-4 and 5-6 represent an application of the obtained results to a sample Weyl semimetal and to the quark-gluon plasma, respectively. The summary is presented in Sec. VII.

\section{MAXWELL-CHERN-SIMONS EFFECTIVE THEORY}

The $C P$-odd domains in the chiral matter can be described by a pseudoscalar field $\theta$ whose interaction with the electromagnetic field $F^{\mu \nu}$ is governed by the Lagrangian $^{1}[16-19]$

\footnotetext{
${ }^{1}$ Throughout the paper the natural rationalized units $\hbar=c=1, \alpha=e^{2} / 4 \pi \approx 1 / 137$ are used.
} 


$$
\mathcal{L}=-\frac{1}{4} F_{\mu \nu}^{2}-\frac{c_{A}}{4} \theta \tilde{F}_{\mu \nu} F^{\mu \nu}+\bar{\psi}\left(i \gamma^{\mu} D_{\mu}-m\right) \psi,
$$

where $\tilde{F}_{\mu \nu}=\frac{1}{2} \epsilon_{\mu \nu \lambda \rho} F^{\lambda \rho}$ is the dual field tensor and $c_{A}=N_{c} \sum_{f} q_{f}^{2} e^{2} / 2 \pi^{2}$ is the chiral anomaly coefficient. In many applications, such as the Weyl semimetals, $\theta$ is time-independent and has constant spatial gradient $\boldsymbol{\nabla} \theta=\boldsymbol{b} / c_{A}$. The same model can describe the spatially inhomogeneous $C P$-odd domains in the quark-gluon plasma [20]. We will use this model throughout the paper. The field equations of electrodynamics in chiral electrically neutral and nonconducting matter read

$$
\begin{aligned}
& \boldsymbol{\nabla} \cdot \boldsymbol{B}=0, \quad \boldsymbol{\nabla} \cdot \boldsymbol{E}=-\boldsymbol{b} \cdot \boldsymbol{B}, \\
& \boldsymbol{\nabla} \times \boldsymbol{E}=-\partial_{t} \boldsymbol{B}, \quad \boldsymbol{\nabla} \times \boldsymbol{B}=\partial_{t} \boldsymbol{E}+\boldsymbol{b} \times \boldsymbol{E} .
\end{aligned}
$$

The monochromatic Fourier components of the electric and magnetic fields satisfy the equations [21]

$$
\begin{aligned}
& \boldsymbol{\nabla} \cdot \boldsymbol{B}=0, \quad \boldsymbol{\nabla} \cdot \boldsymbol{D}=0 \\
& \boldsymbol{\nabla} \times \boldsymbol{E}=i \omega \boldsymbol{B}, \quad \boldsymbol{\nabla} \times \boldsymbol{B}=-i \omega \boldsymbol{D} .
\end{aligned}
$$

where the displacement field $D_{i}=\varepsilon_{i j} E_{j}$ is given by

$$
\boldsymbol{D}=\boldsymbol{E}+\frac{i}{\omega} \boldsymbol{b} \times \boldsymbol{E}=0 .
$$

Equations (4) and (5) describe free electromagnetic field in an anisotropic matter with the dielectric tensor

$$
\varepsilon_{i j}=\delta_{i j}-i \epsilon_{i j k} b_{k} / \omega .
$$

Generally, the dispersive matter supports the transverse and longitudinal electromagnetic waves. However, since the dielectric tensor (7) does not depend on $\boldsymbol{k}$, there is no spatial dispersion and hence no longitudinal waves. The frequencies of the transverse waves can be found using the Fresnel equation

$$
\left|k_{i} k_{j}-k^{2} \delta_{i j}+\omega_{k \lambda}^{2} \varepsilon_{i j}\left(\omega_{k \lambda}\right)\right|=0 .
$$

Substituting (7) into (8) one finds [using e.g., (18)]

$$
\omega_{\boldsymbol{k} \lambda}^{2}=k^{2}+\frac{b^{2}}{2}-\lambda \operatorname{sgn}(\boldsymbol{b} \cdot \boldsymbol{k}) \sqrt{\frac{b^{4}}{4}+(\boldsymbol{b} \cdot \boldsymbol{k})^{2}},
$$

where $\lambda= \pm 1$ is the right/left-handed photon polarizations. ${ }^{2}$ The corresponding group velocity is

\footnotetext{
${ }^{2}$ In the limit $k \ll b$, the dispersion relation (9) has a gapped $\omega_{k_{-}}=b$ and a gapless $\omega_{k+}=k \sin \beta$ branches, where $\cos \beta=$ $\hat{\boldsymbol{b}} \cdot \hat{\boldsymbol{k}}$ (assumed to be positive). Only when $\beta=0$, one recovers the "nonrelativistic photon" $\omega_{k+}=k^{2} / b$ reported in [22].
}

$$
v_{k \lambda}=\frac{\partial \omega_{k \lambda}}{\partial k}=\frac{k}{\omega_{k \lambda}}\left(1+\frac{(\hat{\boldsymbol{k}} \cdot \boldsymbol{b})^{2}}{\omega_{\boldsymbol{k} \lambda}^{2}-k^{2}-b^{2} / 2}\right) .
$$

It is clearly different for the two photon polarizations.

\section{PHOTON WAVE FUNCTION IN ANISOTROPIC DISPERSIVE MEDIUM}

The electromagnetic field in anisotropic dispersive medium was quantized in [23]. The corresponding expression in the radiation gauge is

$$
\boldsymbol{A}(\boldsymbol{x}, t)=\sum_{\boldsymbol{k} \lambda}\left(a_{\boldsymbol{k} \lambda} \boldsymbol{A}_{\boldsymbol{k} \lambda}+a_{\boldsymbol{k} \lambda}^{\dagger} \boldsymbol{A}_{\boldsymbol{k} \lambda}^{*}\right)+\sum_{\boldsymbol{k} \nu}\left(a_{\boldsymbol{k} \nu} \boldsymbol{A}_{\boldsymbol{k} \lambda}+a_{\boldsymbol{k} \nu}^{\dagger} \boldsymbol{A}_{\boldsymbol{k} \lambda}^{*}\right) .
$$

where $\lambda$ runs over the transverse polarizations whereas $\nu$ over the longitudinal ones. The corresponding wave functions read

$$
\begin{aligned}
& \boldsymbol{A}_{\boldsymbol{k} \lambda}=\boldsymbol{e}_{\boldsymbol{k} \lambda}\left(\frac{k v_{\boldsymbol{k} \lambda}}{2 \omega_{\boldsymbol{k} \lambda}^{2} \varepsilon_{i j} e_{k \lambda i}^{*} e_{\boldsymbol{k} \lambda j} V}\right)^{1 / 2} e^{i \boldsymbol{k} \cdot \boldsymbol{x}-i \omega_{k \lambda} t}, \\
& \boldsymbol{A}_{\boldsymbol{k} \nu}=\hat{\boldsymbol{k}}\left(\frac{k^{2}}{\omega_{\boldsymbol{k} \nu}^{2} k_{i} k_{j} \partial \varepsilon_{i j} / \partial \omega_{\boldsymbol{k} \nu} V}\right)^{1 / 2} e^{i \boldsymbol{k} \cdot \boldsymbol{x}-i \omega_{k \nu} t} .
\end{aligned}
$$

The creation and annihilation operators in (11) satisfy the usual bosonic commutation relations

$$
\left[a_{k \lambda}, a_{k^{\prime} \lambda^{\prime}}^{\dagger}\right]=\delta_{k k^{\prime}} \delta_{\lambda \lambda^{\prime}}, \quad\left[a_{k \nu}, a_{k^{\prime} \nu^{\prime}}^{\dagger}\right]=\delta_{k k^{\prime}} \delta_{\nu \nu^{\prime}}, \quad\left[a_{k \lambda}, a_{k^{\prime} \nu}^{\dagger}\right]=0 .
$$

As indicated in Sec. II, a matter with the dielectric tensor (7) does not support the longitudinal waves. Therefore the second term in (11), which is the vector potential operator of the longitudinal waves, vanishes.

As for the transverse waves, for a given photon momentum $\boldsymbol{k}$, the transverse polarization vectors obey the system of equations

$$
\left[k_{i} k_{j}-k^{2} \delta_{i j}+\omega_{\boldsymbol{k} \lambda}^{2} \varepsilon_{i j}\left(\omega_{k \lambda}\right)\right] e_{k \lambda j}=0 .
$$

They has nontrivial solutions labeled by $\lambda$ only if the Fresnel equation (9) is satisfied. The transverse polarization vectors $\boldsymbol{e}_{\boldsymbol{k} \lambda}$ satisfy the following conditions

$$
\begin{aligned}
& \varepsilon_{i j} k_{i} e_{\boldsymbol{k} \lambda j}=0, \\
& \varepsilon_{i j} k_{i} e_{\boldsymbol{k} \lambda i}^{*} e_{\boldsymbol{k} \lambda^{\prime} j}=\varepsilon_{i j} k_{i} e_{\boldsymbol{k} \lambda i}^{*} e_{\boldsymbol{k} \lambda j} \delta_{\lambda \lambda^{\prime}},
\end{aligned}
$$

instead of the usual $\boldsymbol{k} \cdot \boldsymbol{e}_{\boldsymbol{k} \lambda}=0$ and $\boldsymbol{e}_{\boldsymbol{k} \lambda} \cdot \boldsymbol{e}_{\boldsymbol{k} \lambda^{\prime}}^{*}=\delta_{\lambda \lambda^{\prime}}$. Equation (16) indicates that the displacement field $\boldsymbol{D}$ is orthogonal to the wave vector $\boldsymbol{k}$, whereas generally $\boldsymbol{E} \cdot \boldsymbol{k} \neq 0$. 
To find the explicit from of the polarization vectors, consider a Cartesian frame with $z$-axis pointing in the direction of $\boldsymbol{b}$. The dielectric tensor (7) takes form

$$
\varepsilon=\left(\begin{array}{ccc}
1 & -i b / \omega_{k \lambda} & 0 \\
i b / \omega_{k \lambda} & 1 & 0 \\
0 & 0 & 1
\end{array}\right) .
$$

Its eigenvalues are $1 \pm b / \omega$ and 1 with the corresponding eigenvectors - the principal dielectric directions - given by $\hat{\boldsymbol{x}}_{1}=(\hat{\boldsymbol{x}}+i \hat{\boldsymbol{y}}) / \sqrt{2}, \hat{\boldsymbol{x}}_{2}=(\hat{\boldsymbol{y}}+i \hat{\boldsymbol{x}}) / \sqrt{2}$ and $\hat{\boldsymbol{x}}_{3}=\hat{\boldsymbol{z}}$. To diagonalize the dielectric tensor we transform it to the "principal coordinate system" span by these eigenvectors. Transformation matrix $P$ is given by

$$
P=\left(\begin{array}{ccc}
1 / \sqrt{2} & i / \sqrt{2} & 0 \\
i / \sqrt{2} & 1 / \sqrt{2} & 0 \\
0 & 0 & 1
\end{array}\right) .
$$

One can verify that $\tilde{\varepsilon}=P^{-1} \varepsilon P$ is diagonal. In the principal coordinate system (15) reads [21]

$$
\left[k_{i}^{*} k_{j}-k^{2} \delta_{i j}+\omega_{\boldsymbol{k} \lambda}^{2} \tilde{\varepsilon}_{i j}\left(\omega_{\boldsymbol{k} \lambda}, \boldsymbol{k}\right)\right] \tilde{e}_{\boldsymbol{k} \lambda j}=0,
$$

where $i, j=1,2,3$ label the orthogonal directions and we defined $k_{1}=\left(k_{x}-i k_{y}\right) / \sqrt{2}, \quad k_{2}=\left(k_{y}-i k_{x}\right) / \sqrt{2}$, $k_{3}=k_{z}$. In particular, $k^{2}=k_{1}^{*} k_{1}+k_{2}^{*} k_{2}+k_{3}^{2}$. The frequencies $\omega_{k \lambda}$ are of course still given by (9). The corresponding set of polarization vectors is

$$
\tilde{\boldsymbol{e}}_{\boldsymbol{k} \lambda}=C_{\boldsymbol{k} \lambda}\left(\begin{array}{c}
\frac{k_{1} / k}{\left(k / \omega_{k \lambda}\right)^{2}-1-b / \omega_{k \lambda}} \\
\frac{k_{2} / k}{\left(k / \omega_{k \lambda}\right)^{2}-1+b / \omega_{k \lambda}} \\
\frac{k_{3} / k}{\left(k / \omega_{k \lambda}\right)^{2}-1}
\end{array}\right) .
$$

The polarization vectors in (12) are normalized so that $\tilde{\boldsymbol{e}}_{\boldsymbol{k} \lambda}^{*} \cdot \tilde{\boldsymbol{e}}_{\boldsymbol{k} \lambda}=1$. This fixes the normalization constant $C_{\boldsymbol{k} \lambda}$. We won't need the explicit expression for $C_{k \lambda}$ as it cancels out in the final expression for the photon spectrum. The polarization vectors in the original coordinate system are $e_{k \lambda}=P \tilde{e}_{k \lambda}$.

\section{RADIATION RATE}

The scattering matrix element for the transverse photon radiation $f(p) \rightarrow f\left(p^{\prime}\right)+\gamma(k)$ by a fermion of mass $m$ and electric charge $Q$ is given by

$$
S=-i e Q \int \bar{\psi}_{p^{\prime} s^{\prime}} A_{k \lambda}^{*} \psi_{p s} d^{4} x .
$$

Substituting the transverse photon wave function (12) and the electron wave function

$$
\psi_{p s}(\boldsymbol{x}, t)=\frac{1}{\sqrt{2 \varepsilon V}} u_{p s} e^{i p \cdot x-i \varepsilon t},
$$

where $\varepsilon=\sqrt{p^{2}+m^{2}}$ one obtains

$$
\begin{aligned}
S= & -i e Q(2 \pi)^{4} \delta\left(\omega+\varepsilon^{\prime}-\varepsilon\right) \delta\left(\boldsymbol{k}+\boldsymbol{p}^{\prime}-\boldsymbol{p}\right) \\
& \times \frac{\bar{u}_{p^{\prime} s^{\prime}} \phi_{k \lambda}^{*} u_{p s}}{\sqrt{8 \varepsilon \varepsilon^{\prime} \omega V^{3}}}\left(\frac{k v_{k \lambda}}{\omega \varepsilon_{i j} e_{k \lambda i}^{*} e_{k \lambda j}}\right)^{1 / 2},
\end{aligned}
$$

where we employed a shorthand notation $\omega=\omega_{k \lambda}$ and $\varepsilon^{\prime}=\sqrt{p^{\prime 2}+m^{2}}$. The radiation probability can be computed as

$$
d w=\frac{1}{2} \sum_{\lambda s s^{\prime}}|S|^{2} \frac{V d^{3} p^{\prime}}{(2 \pi)^{3}} \frac{V d^{3} k}{(2 \pi)^{3}},
$$

implying that the rate is

$$
\begin{aligned}
d W= & \frac{1}{2(2 \pi)^{2}} e^{2} Q^{2} \sum_{\lambda} \delta\left(\omega+\varepsilon^{\prime}-\varepsilon\right) \delta\left(\boldsymbol{k}+\boldsymbol{p}^{\prime}-\boldsymbol{p}\right) \\
& \times \frac{1}{8 \varepsilon \varepsilon^{\prime} \omega} \frac{k v_{\boldsymbol{k} \lambda}}{\omega \varepsilon_{i j} e_{\boldsymbol{k} \lambda i}^{*} e_{\boldsymbol{k} \lambda j}} \sum_{s s^{\prime}}\left|\mathcal{M}_{0}\right|^{2} d^{3} p^{\prime} d^{3} k,
\end{aligned}
$$

where

$$
\begin{aligned}
\sum_{s s^{\prime}}\left|\mathcal{M}_{0}\right|^{2} & =\operatorname{Tr}\left[(\not p+m) \phi_{\boldsymbol{k} \lambda}^{*}\left(\not \not^{\prime}+m\right) \phi_{\boldsymbol{k} \lambda}\right] \\
& =4 e_{\boldsymbol{k} \lambda i}^{*} e_{\boldsymbol{k} \lambda j}\left[p_{i} p_{j}^{\prime}+p_{j} p_{i}^{\prime}+\delta_{i j}\left(\varepsilon \varepsilon^{\prime}-\boldsymbol{p} \cdot \boldsymbol{p}^{\prime}-m^{2}\right)\right] .
\end{aligned}
$$

The spectrum of photons emitted in a solid angle $d \Omega$ is

$$
\frac{d W}{d \Omega d \omega}=\frac{\alpha Q^{2}}{16 \pi} \sum_{\lambda} \delta\left(\omega+\varepsilon^{\prime}-\varepsilon\right) \frac{k^{3}}{\varepsilon \varepsilon^{\prime} \omega^{2} \varepsilon_{i j} e_{k \lambda i}^{*} e_{k \lambda j}} \sum_{s s^{\prime}}\left|\mathcal{M}_{0}\right|^{2} .
$$

\section{HIGH ENERGY LIMIT}

In practical applications it is useful to consider the high energy limit of the photon spectrum. The asymptotic form of the polarization vectors is obtained by expanding (21) in the limit $b \ll k_{z}$. One finds in the principle coordinate system

$$
\tilde{\boldsymbol{e}}_{\boldsymbol{k} \lambda}=\sqrt{\frac{k^{2}-k_{z}^{2}}{2 k^{2}}}\left(\begin{array}{c}
\frac{k_{1}}{k_{z}-\lambda k} \\
\frac{k_{2}}{k_{z}+\lambda k} \\
1
\end{array}\right) .
$$

One can verify that, up to the terms proportional to the inhomogeneity parameter $\boldsymbol{b}, \tilde{\boldsymbol{e}}_{\boldsymbol{k} \lambda} \cdot \boldsymbol{k}=0$ as it must be in a homogenous matter. Transforming back to the original coordinate system yields

$$
\boldsymbol{e}_{\boldsymbol{k} \lambda}=P \tilde{\boldsymbol{e}}_{\boldsymbol{k} \lambda}=\sqrt{\frac{k^{2}-k_{z}^{2}}{2 k^{2}}}\left(\begin{array}{c}
\frac{k_{x} k_{z}-i \lambda k k_{y}}{k_{z}^{2}-k^{2}} \\
\frac{k_{z} k_{y}+i \lambda k k_{x}}{k_{z}^{2}-k^{2}} \\
1
\end{array}\right) .
$$


Taking now, e.g., $k \approx k_{x} \gg k_{y}, k_{z}$ one obtains $\boldsymbol{e}_{\boldsymbol{k} \pm} \approx$ $\boldsymbol{\epsilon}_{\boldsymbol{k} \pm}=(\hat{z} \mp i \hat{\boldsymbol{y}}) / \sqrt{2}$, i.e., a pair of mutually orthonormal circular polarization vectors. Using the relation $\epsilon_{k \pm}^{*}=\epsilon_{k \mp}$ and the fact that the expression in the square brackets in (27) is symmetric in $i, j$, one obtains in the high energy limit

$$
\begin{aligned}
e_{\boldsymbol{k} \pm i}^{*} e_{\boldsymbol{k} \pm j} & \rightarrow \frac{1}{2}\left(\epsilon_{\boldsymbol{k} \pm i}^{*} \epsilon_{\boldsymbol{k} \pm j}+\epsilon_{\boldsymbol{k} \pm j}^{*} \epsilon_{\boldsymbol{k} \pm i}\right) \\
& =\frac{1}{2}\left(\epsilon_{\boldsymbol{k} \pm i}^{*} \epsilon_{\boldsymbol{k} \pm j}+\epsilon_{\boldsymbol{k} \mp i}^{*} \epsilon_{\boldsymbol{k} \mp j}\right)=\frac{1}{2}\left(\delta^{i j}-\frac{k_{i} k_{j}}{k^{2}}\right) .
\end{aligned}
$$

Substitution into (27) yields

$$
\sum_{s s^{\prime}}\left|\mathcal{M}_{0}\right|^{2}=4\left[\varepsilon \varepsilon^{\prime}-m^{2}-\frac{(\boldsymbol{k} \cdot \boldsymbol{p})\left(\boldsymbol{k} \cdot \boldsymbol{p}^{\prime}\right)}{k^{2}}\right] .
$$

In view of (31) and since $\varepsilon_{i j} e_{k \lambda i}^{*} e_{k \lambda j} \approx 1$, the dependence of the photon radiation rate (28) on $\boldsymbol{b}$ arises entirely from the energy conserving delta-function.

Let $\boldsymbol{n}$ be a unit vector in the direction of the incident momentum: $\boldsymbol{p}=p \boldsymbol{n}, k_{n}=\boldsymbol{k} \cdot \boldsymbol{n}$. In the high energy limit $k_{\perp}, \mu \ll k_{n}$ and $p_{\perp}^{\prime}, m \ll p_{n}^{\prime}$, where $\mu^{2} \approx-\lambda \omega b \cos \beta$ [see (35) below] and $\boldsymbol{k}_{\perp} \cdot \boldsymbol{n}=\boldsymbol{p}_{\perp}^{\prime} \cdot \boldsymbol{n}=0$. Expanding

$k_{n} \approx \omega\left(1-\frac{k_{\perp}^{2}+\mu^{2}}{2 \omega^{2}}\right), \quad p_{n}^{\prime} \approx \varepsilon^{\prime}\left(1-\frac{p_{\perp}^{\prime 2}+m^{2}}{2 \varepsilon^{\prime 2}}\right)$

and using the notation $x=\omega / \varepsilon$ one derives

$$
\sum_{s s^{\prime}}\left|\mathcal{M}_{0}\right|^{2}=\frac{2}{x^{2}(1-x)}\left[k_{\perp}^{2}\left(2-2 x+x^{2}\right)+m^{2} x^{4}\right]
$$

In the same approximation the delta-function can be written as

$$
\delta\left(\omega+\varepsilon^{\prime}-\varepsilon\right) \approx 2 x(1-x) \varepsilon \delta\left(k_{\perp}^{2}+\mu^{2}(1-x)+m^{2} x^{2}\right),
$$

where the photon emission angle is, see Fig. 1,

$$
\cos \vartheta=\frac{\boldsymbol{k} \cdot \boldsymbol{p}}{k p} \approx 1-\frac{k_{\perp}^{2}}{2 x k p},
$$

so that $\vartheta \approx k_{\perp} / \omega$. Using these equations in (28) yields

$$
\begin{aligned}
\frac{d W}{d \Omega d \omega}= & \frac{\alpha Q^{2} x}{2 \pi} \delta\left(x^{2} \varepsilon^{2} \vartheta^{2}+\kappa_{\lambda}\right) \\
& \times\left[\lambda \varepsilon b \cos \beta\left(1-x+\frac{x^{2}}{2}\right)-m^{2} x\right] \theta\left(-\kappa_{\lambda}\right),
\end{aligned}
$$

where $\theta$ is the step function and

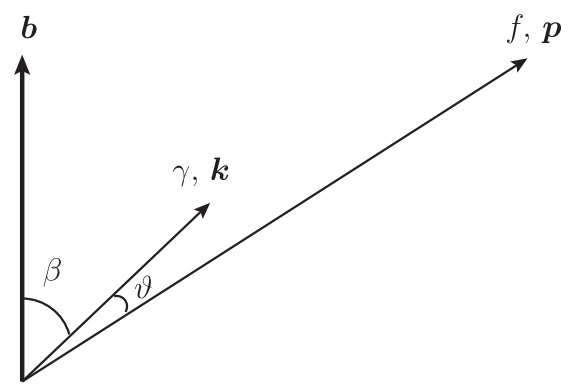

FIG. 1. The geometry of the photon radiation. Since $\vartheta \approx k_{\perp} / \omega \ll 1$, the incident fermion and photon direction $\beta$ with respect to $\boldsymbol{b}$ is almost the same.

$$
\kappa_{\lambda}=\mu^{2}(1-x)+m^{2} x^{2}=-x(1-x) \lambda \varepsilon b \cos \beta+m^{2} x^{2} .
$$

The parameter $\kappa_{\lambda}$ is negative if the following two conditions are satisfied: $\lambda \cos \beta>0$ and $x<x_{\max }$ where

$$
x_{\max }=\left(1+\frac{m^{2}}{\lambda \varepsilon b \cos \beta}\right)^{-1} .
$$

Thus, the photon polarization depends on the incidence angle, which is approximately the same as the angle $\beta$ between the photon direction and $\boldsymbol{b}$ : at $0<\beta<\pi / 2$ it is right-handed, while at $-\pi / 2<\beta<0$ it is left-handed. The spectrum of the right-handed photons at a given incidence angle $\beta$ is the same as that of the left-handed ones at the incidence angle $\pi-\beta$.

To illustrate the main features of the photon spectrum, I will choose a semimetal reported in [24,25] that has $b=(\alpha / \pi) 80 \mathrm{eV}$. The photon radiation rate in a unit solid angle per unit frequency interval (37) is exhibited in Fig. 2 as a function of the emission angle. The sharp peaks correspond to the emission angles of photon with different frequencies and are located at

$$
\vartheta_{0}=\frac{\sqrt{-\kappa_{\lambda}}}{\omega} \approx \sqrt{\frac{\lambda b \cos \beta}{\omega}}
$$

for small enough $x$.

Integration over the solid angle $d \Omega=\pi d \vartheta^{2}$ produces the energy spectrum

$$
\begin{aligned}
\frac{d W}{d x}= & \frac{\alpha Q^{2}}{4 \varepsilon} \frac{1}{x^{2}(1-x)}\left[-\left(\mu^{2}(1-x)+m^{2} x^{2}\right)\left(2-2 x+x^{2}\right)\right. \\
& \left.+m^{2} x^{4}\right] \theta\left(-\kappa_{\lambda}\right) \\
= & \frac{\alpha Q^{2}}{2 \varepsilon x}\left[\lambda \varepsilon b \cos \beta\left(1-x+\frac{x^{2}}{2}\right)-m^{2} x\right] \theta\left(-\kappa_{\lambda}\right)
\end{aligned}
$$

in agreement with the earlier results $[14,15]$. It is plotted in Fig. 3 for different incident angles $\beta$. The spectrum is 


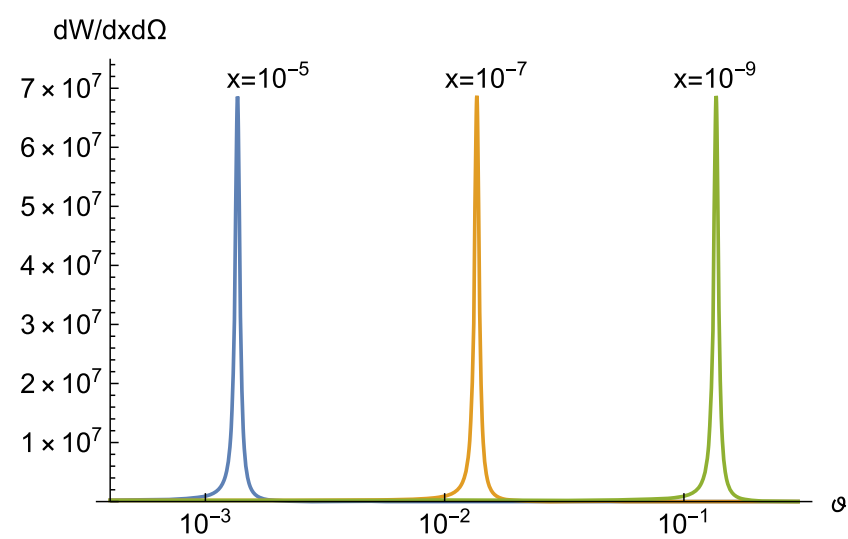

FIG. 2. Angular distribution of the right-hand photons with energies $\omega=x \varepsilon$ emitted by electron of energy $\varepsilon=$ $10 \mathrm{GeV}$ moving through a chiral material with $b=0.19 \mathrm{eV}$ parallel to $\boldsymbol{b}(\beta=0)$. The left-hand photons are not emitted. The delta-function in (37) is approximated by a Lorentzian of width $0.01 \omega$. The maximum possible photon energy fraction is $x_{\max }=7.4 \times 10^{-3}$, see (39).

dominated by soft photons $x \ll 1$ and terminates at $x=x_{\max }$, which varies with $\beta$.

The rate of photon emission into a solid angle is obtained by integrating (37) over the photon energies ${ }^{3}$

$\frac{d W}{d \Omega}=\frac{\alpha Q^{2} x_{0}}{2 \pi \lambda b \cos \beta}\left[\lambda \varepsilon b \cos \beta\left(1-x_{0}+\frac{x_{0}^{2}}{2}\right)-m^{2} x_{0}\right] \theta\left(-\kappa_{\lambda}\right)$,

where the emitted photon energy is fixed at

$$
x_{0}=\left(1+\frac{m^{2}+\varepsilon^{2} \vartheta^{2}}{\lambda \varepsilon b \cos \beta}\right)^{-1} .
$$

One can verify that the rate $(42)$ is proportional to $\left(m^{2}+\right.$ $\left.\varepsilon^{2} \vartheta^{2}+b \varepsilon \lambda \cos \beta\right)^{-3}$ implying that most of radiation is emitted into a cone with the opening angle

$$
\vartheta<\sqrt{\frac{b \varepsilon \lambda \cos \beta+m^{2}}{\varepsilon^{2}}} .
$$

Since for the parameters of the benchmark model $b \varepsilon \ll m^{2}$, the radiation is emitted into a cone with the opening angle $\vartheta<m / \varepsilon$. This is shown in Fig. 4.

As a different example, consider electromagnetic radiation by an ultrarelativistic $u$-quark in the quark-gluon plasma. The spatial gradients of $\theta$ can be reasonably estimated to be of order $\pi / R \sim 1 \mathrm{GeV}$, where $R$ is domain linear size. The corresponding spectra are shown in Fig. 5.

\footnotetext{
${ }^{3}$ Assuming that (9) holds throughout the entire photon spectrum.
}

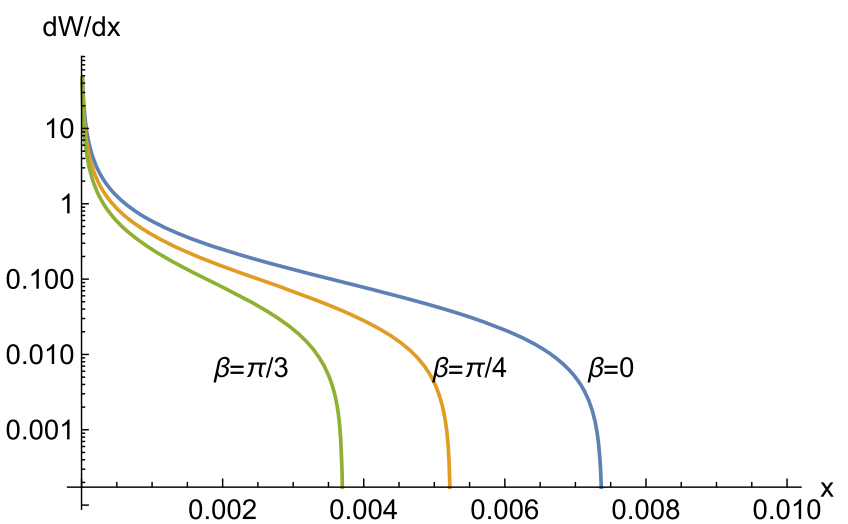

FIG. 3. Spectra of the right-handed photons radiated by electron of energy $10 \mathrm{GeV}$ moving through a chiral material with $b=0.19 \mathrm{eV}$ at different angles to $\boldsymbol{b}$. The same exactly spectra for left-handed photons are obtained at angles $\beta=$ $2 \pi / 3,3 \pi / 4, \pi$ (left to right).

The total photon radiation rate is derived by integrating (41) over $x$. The upper limit of integration is $x_{\max }$ that ensures that $\kappa_{\lambda}<0$. The lower limit $x_{\min }$ can be determined by recalling that the high energy approximation employed in this section assumes that $\omega \gg|\mu|$. This implies that

$$
x_{\min }=\frac{b|\lambda \cos \beta|}{\varepsilon} .
$$

One therefore obtains in the logarithmic approximation (i.e., in the limit $x \ll 1$ )

$$
\begin{aligned}
W & =\int_{x_{\min }}^{x_{\max }} d x \frac{d W}{d x} \\
& =\frac{1}{2} \alpha Q^{2} b|\lambda \cos \beta| \ln \left(\frac{m^{2}}{\varepsilon^{2}}+\frac{b|\lambda \cos \beta|}{\varepsilon}\right)^{-1} .
\end{aligned}
$$

The rate is only logarithmically dependent on the fermion energy $\varepsilon$.

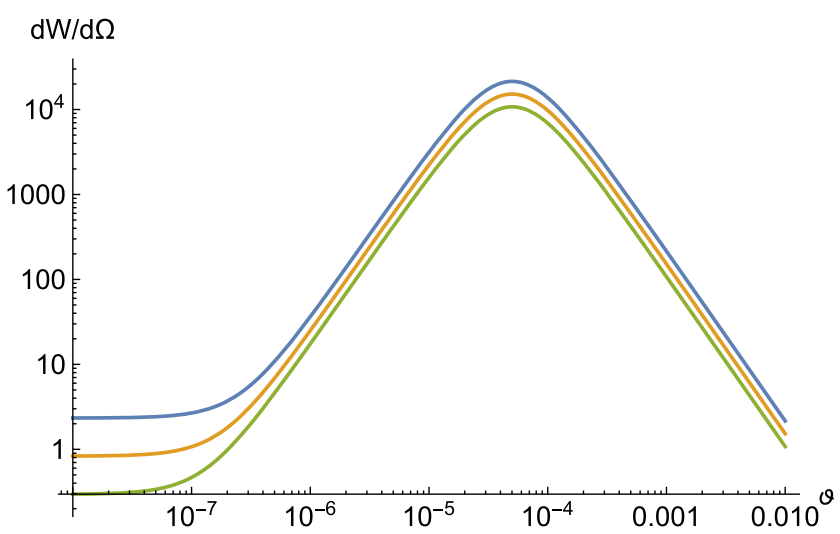

FIG. 4. Angular distribution of the photon radiation produced by electron of energy $10 \mathrm{GeV}$ moving through a chiral material with $b=0.19 \mathrm{eV}$ at the same angles $\beta$ to $\boldsymbol{b}$ as in Fig. 3. The maximum is at $\vartheta \approx m / \varepsilon$. 


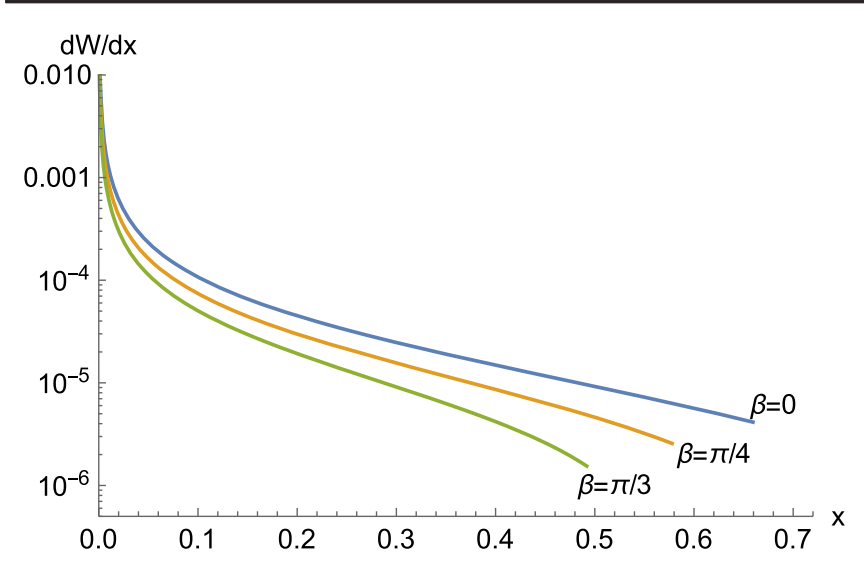

(a)

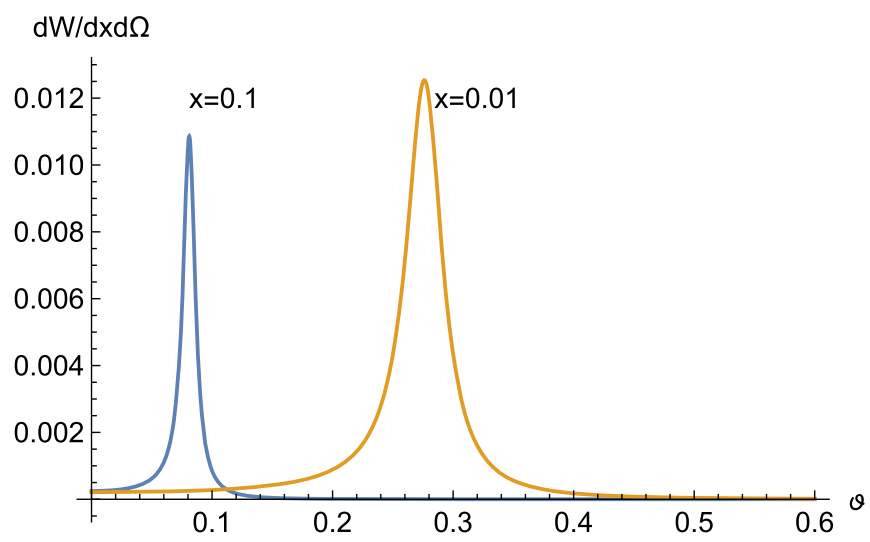

(b)

FIG. 5. Photon spectrum produced by a $u$-quark of energy $10 \mathrm{GeV}$ and thermal mass $0.2 \mathrm{GeV}$ moving in quark-gluon plasma. (a) Integrated over the photon emission angle for different incident angles $\beta$. (b) Angular distribution of the radiation for a fixed photon energy. In both plots the radiation is right-hand polarized.

\section{TRANSITION RADIATION}

When a fermion radiating the electromagnetic radiation crosses the boundary between the chiral matter and vacuum, it emits the chiral transition radiation discussed recently in $[14,15]$. Assuming that the fermion is incident at the normal angle to the boundary and that there are no surface currents on it, the spectrum of the radiated photons of a given polarization $\lambda$ is given by

$$
\begin{aligned}
\frac{d N_{\lambda}}{d^{2} k_{\perp} d x}= & \frac{\alpha Q^{2}}{2 \pi^{2} x}\left\{\left(\frac{x^{2}}{2}-x+1\right) k_{\perp}^{2}+\frac{x^{4} m^{2}}{2}\right\} \\
& \times\left[\frac{1}{k_{\perp}^{2}+\kappa_{\lambda}}-\frac{1}{k_{\perp}^{2}+m^{2} x^{2}}\right]^{2},
\end{aligned}
$$

which coincides with the spectrum of the ordinary transition radiation when $\kappa_{\lambda}>0$ [26,27]. The spectrum (47) has a resonance at $k_{\perp}=-\kappa_{\lambda}$ corresponding to the delta-

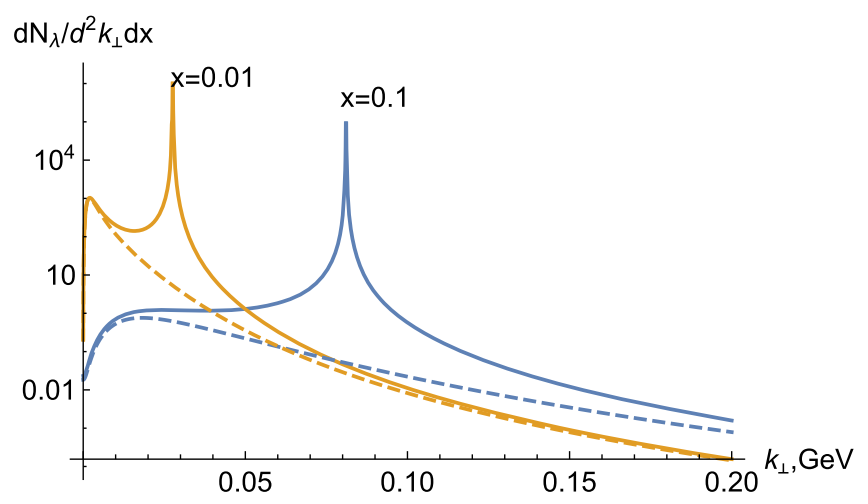

FIG. 6. Photon spectrum emitted by a $u$-quark of energy $10 \mathrm{GeV}$ and thermal mass $0.2 \mathrm{GeV}$ moving in quark-gluon plasma at $\beta=0$. Solid lines: right-handed photons, dashed lines: left-handed photons. function in (37). Thus, the formulas for the spectra of the photon radiation derived in the previous section describe this resonant behavior. In Fig. 6 one can see both the Cherenkov radiation peaks, which are the same as the peaks seen on Fig. 5(b), and the transition radiation continuum. Unlike the chiral Cherenkov radiation, the chiral transition radiation is a boundary effect.

\section{SUMMARY}

The most important features of the photon spectrum observed in Figs. 2-6 are

(i) The angular distribution $d W / d \Omega d \omega$ peaks at certain emission angles $\vartheta_{0}$ that depends on photon energy $\omega$ and direction $\beta$ with respect to $\boldsymbol{b}$, in agreement with the earlier observations $[6,7]$.

(ii) The spectrum is always circularly polarized; the polarization direction (right or left) depends on whether the angle $\beta$ is acute of obtuse.

(iii) The spectrum $d W / d x$ terminates when the photon carries the maximum allowed fraction $x_{\max }$, given by (39), of the fermion energy.

(iv) The total rate is proportional to $b$, but only logarithmically depends on the fermion energy.

I believe that these observations can make possible the experimental measurements of the novel electromagnetic radiation process in chiral materials. In particular, it can prove the existence of the $C P$-odd domains in quark-gluon plasma.

\section{ACKNOWLEDGMENTS}

I am grateful to Igor Shovkovy and Xu-Guang Huang for informative discussions. This work was supported in part by the U.S. Department of Energy under Grant No. DEFG02-87ER40371. 
[1] A. A. Zyuzin and A. A. Burkov, Topological response in Weyl semimetals and the chiral anomaly, Phys. Rev. B 86, 115133 (2012).

[2] A. G. Grushin, Consequences of a condensed matter realization of Lorentz violating QED in Weyl semi-metals, Phys. Rev. D 86, 045001 (2012).

[3] F. R. Klinkhamer and G. E. Volovik, Emergent $C P T$ violation from the splitting of Fermi points, Int. J. Mod. Phys. A 20, 2795 (2005).

[4] D. Kharzeev and A. Zhitnitsky, Charge separation induced by P-odd bubbles in QCD matter, Nucl. Phys. A797, 67 (2007).

[5] D. E. Kharzeev, The chiral magnetic effect and anomalyinduced transport, Prog. Part. Nucl. Phys. 75, 133 (2014).

[6] R. Lehnert and R. Potting, Vacuum Cherenkov Radiation, Phys. Rev. Lett. 93, 110402 (2004).

[7] R. Lehnert and R. Potting, The Cerenkov effect in Lorentzviolating vacua, Phys. Rev. D 70, 125010 (2004); Erratum, Phys. Rev. D 70, 129906(E) (2004).

[8] V. A. Kostelecky and A. G. M. Pickering, Vacuum Photon Splitting in Lorentz Violating Quantum Electrodynamics, Phys. Rev. Lett. 91, 031801 (2003).

[9] D. Mattingly, Modern tests of Lorentz invariance, Living Rev. Relativity 8, 5 (2005).

[10] T. Jacobson, S. Liberati, and D. Mattingly, Lorentz violation at high energy: Concepts, phenomena and astrophysical constraints, Ann. Phys. (Amsterdam) 321, 150 (2006).

[11] B. Altschul, Vacuum Cerenkov Radiation in LorentzViolating Theories without CPT Violation, Phys. Rev. Lett. 98, 041603 (2007).

[12] B. Altschul, Cerenkov radiation in a Lorentz-violating and birefringent vacuum, Phys. Rev. D 75, 105003 (2007).

[13] J. R. Nascimento, E. Passos, A. Y. Petrov, and F. A. Brito, Lorentz-CPT violation, radiative corrections and finite temperature, J. High Energy Phys. 06 (2007) 016.
[14] K. Tuchin, Radiative instability of quantum electrodynamics in chiral matter, Phys. Lett. B 786, 249 (2018).

[15] X. G. Huang and K. Tuchin, Transition Radiation as a Probe of Chiral Anomaly, Phys. Rev. Lett. 121, 182301 (2018).

[16] F. Wilczek, Two Applications of Axion Electrodynamics, Phys. Rev. Lett. 58, 1799 (1987).

[17] S. M. Carroll, G. B. Field, and R. Jackiw, Limits on a Lorentz and parity violating modification of electrodynamics, Phys. Rev. D 41, 1231 (1990).

[18] P. Sikivie, On the interaction of magnetic monopoles with axionic domain walls, Phys. Lett. B 137, 353 (1984).

[19] T. Kalaydzhyan, Chiral superfluidity of the quark-gluon plasma, Nucl. Phys. A913, 243 (2013).

[20] K. Tuchin, Spontaneous topological transitions of electromagnetic fields in spatially inhomogeneous $C P$-odd domains, Phys. Rev. C 94, 064909 (2016).

[21] Z. Qiu, G. Cao, and X. G. Huang, On electrodynamics of chiral matter, Phys. Rev. D 95, 036002 (2017).

[22] N. Yamamoto, Axion electrodynamics and nonrelativistic photons in nuclear and quark matter, Phys. Rev. D 93, 085036 (2016).

[23] A. I. Alekseev and Yu. P. Nikitin, Quantization of the electromagnetic field in a dispersive medium, Sov. Phys. JETP 23, 608 (1966).

[24] S. Y. Xu et al., Discovery of a Weyl Fermion semimetal and topological Fermi arcs, Science 349, 613 (2015).

[25] B. Q. Lv et al., Experimental Discovery of Weyl Semimetal TaAs, Phys. Rev. X 5, 031013 (2015).

[26] V. N. Baier and V. M. Katkov, Quantum theory of transition radiation and transition pair creation, Phys. Lett. A 252, 263 (1999).

[27] D. Schildknecht and B. G. Zakharov, Transition radiation in quantum regime as a diffractive phenomenon, Phys. Lett. A 355, 289 (2006). 